\title{
The Most Informative Mistake about Report Interpretation of Slit Skin Smear
}

\author{
Pugazhenthan Thangaraju* and Sajitha Venkatesan \\ Central leprosy teaching and research institute, India
}

*Corresponding author: Pugazhenthan Thangaraju, MBBS, MD, DNB, MNAMS, Central leprosy teaching and research institute, Ministry of health and family welfare, Government of India, Chengalpattu, India

Submission: 眥 February 09, 2018; Published: 眥 February 16, 2018

\section{Editorial}

As a beginner in leprosy field, we do encounter with mistake of interpretation of results of slit skin Smear (SSS). A skin smear is a diagnostic \& prognostic test in which a sample of material is Collected from a tiny cut in the skin and then stained for M. lepra. This test is done for to confirm clinical diagnosis of multi-bacillary leprosy in a suspect, to determine the state of infectivity, to help in diagnosis of relapse of leprosy, for classification and prognosis and to get negative certificate. The lepra bacilli will be seen as pink rods. The bacilli may be seen as in single, or in small groups or as a closely packed bundle called globi or in any combinations of these. The smears are graded and two parameter namely Bacterial Index (BI) and Morphological Index (MI) are seen. BI is the average number of bacilli per microscopic field and grading done per Ridley Joplings scale. Lepra bacilli when stained by Ziehl-Neelson's method take pink colour stain. But most of the bacilli are stained irregularly and only a few are uniformly stained and the uniformly, intensely stained bacilli are viable and capable of multiplying and that all the other forms are dead or dying and are incapable of multiplying. The percentages of solid bacilli are called MI. The changes in the morphology of human leprosy bacilli serve as useful indicators of progress during treatment. Organisms which reveal such characteristics in their morphology are considered to be nonviable at least in their ability to propagate in another living host. A clinical suspect case of Hansen disease reported to our hospital. We did split skin smear for diagnosis and classification of leprosy. The result came as positive with BI of 4 and MI of $3 \%$. The patient was prescribed MB-MDT (A) comprising Rifampicin, Dapsone and Clofazimine for 12 months as per WHO /NLEP guidelines and advised to come monthly for drugs and consultation.

After 2 months patient presented with increase in size of lesion and fever. As a beginner I thought of relapse and advised for SSS again. The result is positive with BI of 6 and MI of $1 \%$. Since I was with the wrong impression that leprosy is eliminated and nothing to be updated I thought that the patient is not responsive with drugs and suspected primary resistance for available drugs for leprosy and planned for polymerase chain reaction (PCR) and animal study for suspicion of relapse due to drug resistance. But my senior rightly pointed my mistake of interpretation of results. As above it is clearly defined MI is the solid bacilli and once it is killed by antileprosy drugs the solid bacilli will be broken into many fragments that increases the BI as it is the average densities of bacilli (solid and broken, fragmented). In this case the BI increased with reduction in MI proving the susceptibility of drugs. The symptoms of fever and increase in size of patch are due to reaction that is encountered in certain population of leprosy patient. The patient was followed throughout the treatment regimen and at the time of release from treatment (RFT) the patient BI is 2.13 with MI of Zero. From these we also understood that it will take a year minimum for 1 log reduction of BI depends upon the clearing capacity of host immune system. From this case we can learn the proper interpretation of reports and based on that we started practicing and teaching the various faculties and students regarding leprosy and slit skin smear. 
Creative Commons Attribution 4.0

International License

For possible submission use the below is the URL
Your subsequent submission with Crimson Publishers will attain the below benefits

- High-level peer review and editorial services

- Freely accessible online immediately upon publication

- Authors retain the copyright to their work

- Licensing it under a Creative Commons license

- Visibility through different online platforms

- Global attainment for your research

- Article availability in different formats (Pdf, E-pub, Full Text)

- Endless customer service

- Reasonable Membership services

- Reprints availability upon request

- One step article tracking system 